\title{
Social and ethical implications of psychiatric classification for low- and middle-income countries
}

\author{
J K Burns, MB ChB, FCPsych(SA), MSc(Res), PhD \\ Department of Psychiatry, University of KwaZulu-Natal, Durban, South Africa
}

Corresponding author: J K Burns (burns@ukzn.ac.za)

\begin{abstract}
With the publication of the Diagnostic and Statistical Manual of Mental Disorders, 5th edition, and the ongoing revision of the International Classification of Diseases, currently 10th edition, it is timely to consider the wider societal implications of evolving psychiatric classification, especially within low- and middle-income countries (LMICs).

The author reviewed developments in psychiatric classification, especially the move from categorical to dimensional approaches based on biobehavioural phenotypes. While research supports this move, there are several important associated ethical challenges. Dimensional classification runs the risk of 'medicalising' a range of normality; the broadening of some definitions and the introduction of new disorders means more people are likely to attract psychiatric diagnoses. Many LMICs do not have the political, social, legal and economic systems to protect individuals in society from the excesses of medicalisation, thus potentially rendering more citizens vulnerable to forms of stigma, exploitation and abuse, conducted in the name of medicine and psychiatry. Excessive medicalisation within such contexts is also likely to worsen existing disparities in healthcare and widen the treatment gap, as inappropriate diagnosis and treatment of mildly ill or essentially normal people has an impact on health budgets and resources, leading to relative neglect of those with genuine, severe psychiatric disorders.

In an era of evolving psychiatric classification, those concerned for, and involved in, global mental health should be critically self-reflective of all aspects of the modern psychiatric paradigm, especially changes in classification systems, and should alert the global profession to the sociopolitical, economic and cultural implications of changing nosology for LMIC regions of the world.
\end{abstract}

S Afr J Psych 2014;20(3):66-71. DOI:10.7196/SAJP.589

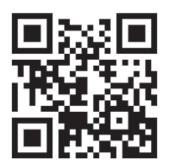

The revision of the two major classification systems in psychiatry, the International Classification of Diseases, $10^{\text {th }}$ edition (ICD-10) and the Diagnostic and Statistical Manual of Mental Disorders, $4^{\text {th }}$ edition, text revised (DSM-IV-TR), is in process; the Diagnostic and Statistical Manual of Mental Disorders, 5th edition (DSM-5) was published in May 2013 and the ICD-11 is expected in 2015. It is therefore an opportune time to consider the wider societal implications of changing psychiatric classification, especially within low- and middle-income country (LMIC) contexts. It is implicit that altering our definitions and concepts of mental disorders will have social, cultural, political, economic, legal and ethical consequences. While the two parent organisations of the respective classification systems - namely the World Health Organization (WHO) for the ICD and the American Psychiatric Association (APA) for the DSM - have made some effort to include experts from so-called developing countries in their revision committees, it is inevitable that the completed ICD-11 and DSM-5 will reflect more closely the perspectives and values of so-called developed countries. Expressed more cynically, it is inevitable that the finished products will reflect the agendas of European and North American psychiatry. This is inevitable and not necessarily bad. However, the fact that both the ICD and DSM systems are globally dominant, and yet emerge almost entirely from the historical traditions and contemporary beliefs of northern hemisphere nations, means that some degree of dissonance is to be expected.
The primary objective of this article is to consider some of the more controversial and radical developments in psychiatric classification, giving specific consideration to the question of how anticipated changes in nosology might affect poorly resourced developing contexts. The author argues that a global mental health (GMH) approach requires a critical, self-reflective attitude to all aspects of the modern psychiatric paradigm, including its classification systems, and consideration of the possible implications of changing these classification systems for the practice of psychiatry and mental healthcare in different social, economic and cultural contexts.

\section{From categories to dimensions}

\section{Problems with categorising mental disorders}

Perhaps the most significant development in psychiatric classification in recent years has been the steady increase in evidence for dimensions of psychopathology that clearly are not accommodated by the categorical approaches of the DSM and ICD systems. ${ }^{[1]}$ Existing systems require the clinician to make a decision as to whether an individual fits into a discrete, well-defined diagnostic category. In ICD-10, prototype descriptions of disorders are provided with diagnostic guidelines, ${ }^{[2]}$ while in DSM-5, lists of criteria are provided; in most cases an individual must have a minimum number of symptoms from a list to qualify for a diagnosis. Both systems are problematic - the DSM system perhaps more so - because in reality, 
individuals often manifest behavioural, emotional and cognitive symptoms that either overlap more than one diagnostic category or are insufficient in quality or quantity to meet diagnostic criteria for a category. For example, grandiosity may be a feature of mania, schizophrenia, narcissistic personality or frontal lobe damage, or indeed may be regarded as normal in certain contexts. The clinician is faced with a diagnostic dilemma when an individual has some features of a specific disorder - e.g. two or three features of major depressive disorder (MDD) - but the required number of symptoms is not present and thus a diagnosis of MDD cannot be made. In DSM-5, where diagnostic criteria are defined in terms of number of symptoms, duration of symptoms and presence of functional impairment, there are a variety of situations whereby an individual misses diagnosis by falling short of a required criterion.

The problems with such a system are numerous. Firstly, in many contexts, failing to attract a diagnosis can have multiple negative consequences. These may include: not accessing appropriate treatment; not receiving medical insurance cover for consultations and treatment; and being culpable before the law. In most highincome countries (HICs) these negative consequences are often mitigated by sophisticated health, welfare and social systems that recognise and acknowledge the importance and relevance of subdiagnostic psychopathology; and processes have been developed to accommodate such individuals. For example, where comprehensive mental health services are plentiful and accessible to all, an individual with subsyndromal depression may be provided with a psychotherapeutic intervention to prevent progression and restore healthy functioning. However, such services are unlikely to be available to individuals without a diagnosis in most LMIC contexts, where mental health resources are often scarce and inaccessible to even the profoundly ill. ${ }^{[3]}$ Similarly, in countries where legal systems are not sensitised to the fact that mental illness can manifest in countless forms and degrees of severity, the absence of a solid diagnosis may prejudice a defendant who is nonetheless compromised by a subdiagnostic mental disorder. Of course, widening diagnostic borders to include more subtle forms of mental ill-health would bring its own challenges to the justice system, where offenders who are not actually psychiatrically ill might be afforded lesser sentences or even acquitted on the basis of minor or indeed feigned symptoms.

The dangers of a strict categorical system are well illustrated in the early stages of psychosis where an individual is deteriorating in function and is beginning to experience occasional or partial symptoms. There is good evidence that these attenuated symptom states often precede the onset of frank psychosis by months or even years. ${ }^{[4]}$ The importance of early intervention with psychosocial and possibly even pharmacological therapies has been demonstrated in multiple studies. Early detection and intervention in psychosis can diminish comorbidities such as substance abuse and depression, hasten response to treatment, reduce relapse and improve overall course and long-term outcome. ${ }^{[5]}$ In many countries, specialised early intervention services are now routine. However, current categorical classification systems do not make provision for allocation of a diagnosis in the early stages of psychosis. In many contexts, especially those with limited resources, the absence of a diagnosis in such cases becomes a barrier to treatment or even preventive interventions. This means that individuals in the early stages of severe mental disorders and residing within LMIC contexts are often deprived of critical services. Without early intervention, the path to recovery is likely to be more difficult. This is a clear example of the inequalities that exist in mental healthcare between HIC and LMIC contexts.

\section{Towards dimensions of psychopathology}

Over the last 20 years, countless neurobiological and clinical research studies have yielded evidence supporting a dimensional approach to the classification of psychopathology. ${ }^{[6]}$ Notably, dimensions or continua exist between disorders and also between disorder and normality. ${ }^{[7]}$ Data from genetics, neuroimaging, neurochemistry and neuropathology point to biological continuity between many disorders that are currently categorically classified. This evidence is challenging assumptions we hold about specific disorders, some of which we have clung to for more than a century. For example, recent advances in genetics (e.g. the investigation of copy number variants) indicate a shared molecular basis for schizophrenia, bipolar disorder, autism and epilepsy. Other genetic studies support biological continuity between schizophrenia and the major mood disorders. ${ }^{[8]}$ These discoveries invalidate and undermine our dichotomous classification of the psychoses along Kraepelinian lines. Neuropsychological features such as theory of mind impairment, initially thought to be specific to autism, have now been demonstrated in a range of disorders including schizophrenia, bipolar disorder and dementia. ${ }^{[9]}$ Mood instability may be a feature of both bipolar disorder and borderline personality disorder, while obsessive and compulsive behaviours transcend several traditional categories, including obsessive compulsive disorder, obsessive compulsive personality disorder, somatoform disorders such as body dysmorphic disorder and hypochondriasis, and impulse control disorders such as trichotillomania and skin picking disorder.

The DSM-5 task team had an express agenda to move towards a dimensional system of classification: ${ }^{[1]}$ however, this has not been achieved and the manual remains a categorical system. However, the move towards dimensional classification in psychiatry will undoubtedly gather momentum in the future, and regular updates of DSM-5 are anticipated over the next decades. ${ }^{[10]}$ Referring to the 'more dimensional DSM-5 approach and organisational structure', the authors of DSM-5 state in their introduction to the manual: 'Such a reformulation of research goals should also keep DSM-5 central to the development of dimensional approaches to diagnosis that will likely supplement or supersede current categorical approaches in coming years. ${ }^{[11]}$ The DSM-ICD Harmonization Group was established to align the respective revision processes, and consequently it is anticipated that ICD-11 will also represent a significant shift towards dimensional classification. ${ }^{[12]}$

\section{Ethical implications of the dimensional approach}

While substantial evidence supports the move towards a dimensional approach to classification, there are a number of important ethical implications of introducing such a system. A dimensional approach runs the risk of medicalising a range of normality as the boundaries between what is considered pathological and what is accepted as normal are pushed back. For example, studies from many countries now show that subsyndromal psychotic-like experiences (PLEs) 
are experienced by up to $10 \%$ of the general population. ${ }^{[13]}$ Most of these individuals are not ill and function normally. While better characterisation of the dimension between non-affective disorders and schizophrenia is likely to progress the field and perhaps have a positive impact on treatment strategies, the recognition of a schizophrenia spectrum from classic chronic psychosis to healthy, well-functioning people with PLEs at the other, may have some undesired results. At what point does normality become pathology? What impact will dimensionality have on society and on our concepts of normality? On the one hand, a dimensional approach could break down stigma in that it implies continuity between pathology and normality and reduces the discrete grouping of individuals, which is a powerful ingredient of stigma. ${ }^{[14]}$ But on the other hand, it could increase stigma for more people as the borderlands of normality become medicalised and labelled as disorders. This highlights what perhaps might be one of psychiatry's most important ethical responsibilities: How do we balance making sure that people get the treatment they need versus increasing medicalisation of our society? In the case of psychosis, this issue is clearly illustrated in the debate surrounding early intervention and the inclusion of a new condition, attenuated psychosis syndrome (APS), in the 'Conditions for further study' chapter of the DSM-5. How do we ensure effective early intervention for incipient psychosis, which may have the effect of radically improving the course and outcome of the disease, and at the same time avoid committing individuals with non-pathological PLEs to unnecessary treatment and the stigma of a psychiatric diagnosis? The key cornerstones of psychiatric classification that continue to be the primary discriminatory factors between normal and pathological are the concepts of distress and level of function. In the case of APS it is reassuring that the proposed diagnostic criteria require the attenuated psychotic-like symptoms to be 'sufficiently distressing and disabling to the individual to warrant clinical attention. ${ }^{[11]}$ One can thus hope that this requirement would serve to protect healthy, well-functioning individuals with PLEs from attracting a psychiatric diagnosis.

As it turns out, the DSM-5 task team elected not to include APS as a disorder in this version of the manual, arguing that the syndrome requires further research. This is perhaps a good thing, especially in LMICs. Whereas the categorical system may result in a lack of access to treatment for individuals falling short of disorder criteria within poorly resourced contexts, a dimensional system in the same context may lead to the inappropriate over-diagnosis and treatment of individuals not meriting treatment, by inexpert healthcare providers. This author has had the personal experience of reviewing the case of a man diagnosed ten years earlier in a community clinic as having schizophrenia and placed on a long-acting, first-generation injectable antipsychotic. The patient had not been reviewed by a psychiatrist for a decade and had tardive dyskinesia. On review, it was clear that his single 3-month illness ten years earlier had been a major depressive episode with transient psychotic symptoms. Sadly, this man's story is not unusual in contexts characterised by major human and infrastructure resource limitations. In a GMH era, where strategies such as task shifting and integration of mental health into primary healthcare are widely advocated, it is important to be wary of unwittingly prescribing 'poor medicine for poor people. There is truth in the saying that 'a little knowledge can be dangerous'; the authors of diagnostic systems such as the DSM and ICD should be mindful of the fact that their decisions made in committee rooms in Europe and North America will dictate the practices of health workers with widely differing levels of knowledge and expertise on far-off continents. Task shifting and integration of mental health into primary care may be appropriate and necessary strategies in contexts where psychiatrists and psychologists simply don't exist, but it is critical that these approaches are planned and implemented together with expert training and supervision. For such interventions to be relevant to local contexts, it is essential that this work is shared through collaborative efforts between both the experts and local stakeholders who often hold key knowledge of what is appropriate and acceptable. This will become even more necessary if we are to be faithful to our dictum primum non nocere, as classification and diagnostic systems evolve and change.

\section{Concerns about changes in the DSM-5}

Some worrying changes have already crept into the new DSM-5, mostly related to relaxation of existing definitions of disorders - broadening concepts of psychopathology so that ultimately individuals who previously fell outside categorical boundaries are now diagnosable and will qualify for treatment. In addition, there are new diagnostic categories, many of which do not have sufficient empirical backing. In early 2012, an open letter was addressed to the DSM-5 taskforce and the APA, endorsed by more than 14000 individuals and over 50 professional organisations. ${ }^{[15]}$ The authors of the open letter argued that 'Increasing the number of people who qualify for a diagnosis may lead to excessive medicalisation and stigmatisation of transitive, even normative distress'. Examples of some of these changes include: the reduction in the number of criteria necessary for the diagnosis of attention deficit hyperactivity disorder; the removal of the major depressive disorder bereavement exclusion, which previously prevented the pathologisation of grief, a normal life process (normal grief will now easily become major depressive disorder); the formalisation of binge eating disorder; and minor cognitive disorders defined as diagnostic categories, which raises concern over the possibility of over-diagnosis. Allen Frances, the chairperson of the DSM-IV taskforce, is a strong critic of what he terms 'diagnostic inflation' within the DSM system: 'The DSMs have introduced many new diagnoses that were no more than severe variants of normal behavior [sic]. ${ }^{[16]}$ Of particular concern is the fact that vulnerable groups, including children and the elderly, are the targets of several diagnostic changes that will widen the net of those fulfilling criteria for pharmacological interventions. The recent National Comorbidity Survey for mental disorders in the USA, which was based on DSM-IV criteria, reported that $50 \%$ of the population will have a psychiatric disorder at some point in their lives. ${ }^{[17]}$ It is yet to be determined to what extent the expanded diagnostic system in DSM-5 would inflate this estimate.

In relation to children, DSM-5 has introduced a new disorder, disruptive mood dysregulation disorder, which must be diagnosed by age 10 years. For a child to attract this diagnosis, he or she must display 'severe recurrent temper outbursts manifested verbally and/ or behaviourally that are grossly out of proportion in intensity or duration to the situation or provocation. ${ }^{\text {[11] }}$ Furthermore, these 
outbursts must occur on average three or more times a week and they must be 'inconsistent with developmental level'. What this implies is that a child prone to regular temper tantrums can now be diagnosed with a psychiatric disorder; which in turn may be accompanied by potential stigma, labelling and psychopharmacological interventions. With regard to the elderly, there is now a new DSM-5 diagnosis called mild neurocognitive disorder. While this may be a useful additional diagnosis in individuals living with HIV or another medical condition that is associated with possible cognitive impairment, the concern here is for otherwise healthy elderly people who begin to show subtle signs of what was previously regarded as normal ageing. Such individuals, according to DSM-5, need only display 'evidence of modest cognitive decline from a previous level of performance in one or more cognitive domains. ${ }^{[11]}$ In addition, as long as there is subjective concern and as long as the 'modest' impairment is documented objectively through clinical assessment, the diagnosis can be made without any evidence of functional impairment. Thus, in practice, it is quite feasible for the onset of normal forgetfulness in an older person to be labelled as a psychiatric disorder, along with all its associated implications.

These two examples illustrate clearly how DSM-5 has relaxed the boundaries of what is considered normal within vulnerable groups such as children and the elderly, where normal developmental change means that these stages are typically quite variable between individuals. By widening the net of what is now considered abnormal, these diagnostic changes are likely to result in substantial numbers of young and old people being subjected to drug treatments. Importantly, the responsible and safe use of these new diagnoses to improve wellbeing of individuals requires experience and a mature understanding of the relative balance between help and harm. In well-resourced contexts and in expert hands, these changes may indeed bring help to those previously denied it. But in large parts of the world where such expertise is not available, there is a considerable risk of harm being done to many people. It is very difficult for the authors of DSM diagnoses to conceptualise the widespread resource gap in psychiatric and psychological care in most LMICs, and to appreciate the dangers within such contexts of over-diagnosis, misdiagnosis and ultimately harmful practice. In this author's view, the decisions by the APA to cancel the second round of DSM-5 field trials and to refuse calls for external peer review of the criteria prior to publication, call into question the utility of the manual in poorly resourced, LMIC contexts.

\section{The dangers of medicalisation within poorly resourced contexts}

All the concerns expressed about medicalisation in psychiatry within well-resourced contexts also apply to poorly resourced contexts, including concerns about increased stigma, increased social and political control, and economic implications. Arguably, however, the conditions of poorly resourced environments amplify the potential dangers of psychiatric medicalisation. This is because these environments usually do not have adequate political, social, legal and economic systems to protect individuals from the excesses of medicalisation. Citizens are thus left vulnerable to potential exploitation and abuse at the hands of societal institutions such as health systems, legal, social and even political entities, as well as the pharmaceutical industry, all occurring in the name of medicine and psychiatry. For example, it is widely known that regulation of the pharmaceutical industry varies considerably between countries, and in many countries, particularly LMICs, the conduct of that industry is virtually unregulated. ${ }^{[18]}$ Globally, many authors have expressed concern at 'the phenomenon of 'disease mongering' ... in the marketing of various drugs (e.g. the selective serotonin re-uptake inhibitors, SSRIs),'[19] especially in relation to conditions such as 'mild social anxiety', described by Lane ${ }^{[20]}$ as the 'medicalisation of shyness'. Within poorly regulated contexts, it is not hard to imagine the potential for gross abuses of the codes of conduct that should govern the relationship between doctors and the pharmaceutical industry. In such scenarios, the ultimate victims of such abuses are the patients who leave consulting rooms with new psychiatric diagnoses and shopping lists of expensive medications. Health practitioners and health services are by no means innocent in such scenarios - managed healthcare in the private sector (with limits on admission periods) and pressure on inpatient facilities due to inadequate resources in the public sector, force patterns of practice that emphasise the imperative to hurry treatment and get patients out of hospital as quickly as possible. Prescribing highdose combination medications becomes the key to coping with high patient volumes for most (usually well-meaning) practitioners. This is not unique to LMIC contexts, but is inevitably more likely to occur where resources for mental healthcare are limited and under pressure. The negative and damaging consequences are felt almost exclusively by patients and their families, who bear the burden of suboptimal and sometimes harmful treatments.

The same concern pertains to the increased potential for abuse of psychiatric labelling and treatment for nefarious social and political means within countries lacking sound mental health and related legislation based on human rights principles. A recent review of mental health systems across 191 countries reported that $92 \%$ of countries in the European region have mental health legislation, compared to only $55 \%$ of countries in the eastern Mediterranean region and $66 \%$ of countries within Africa. ${ }^{[21]}$ It follows that less protection is available within these LMIC regions for those with psychiatric diagnoses, meaning that receiving a psychiatric diagnosis is likely to render the individual vulnerable to potential exploitation and abuse. In relation to inappropriate psychiatric over-diagnosis, Kawa and Giordano $^{[19]}$ state: 'While such categorisations may arise from, and be directed toward, benevolent intentions, caution is required to ensure against sociopolitical usurpation of these diagnoses, and repetition of historical instances of bastardisation of medicine by capricious agenda. The recent history of psychiatry is littered with examples of 'sociopolitical usurpation' and 'bastardisation of medicine' and it is perhaps not surprising that this has tended to happen in societies where the rule of law has become corrupted. Atrocities committed under the guise of psychiatry were commonplace in Nazi Germany and Stalinist USSR, and more recently occurred in apartheid South Africa (SA). In the present day, within contexts characterised by inadequate or even unjust legal frameworks, the psychiatric profession needs to be especially vigilant in safeguarding the human rights of all citizens, both those with clear psychiatric disorders and those who exist on the diagnostic fringes.

The cross-cultural utility of psychiatric diagnostic systems has been the subject of endless discussion and criticism. In a comparison of 
DSM and ICD systems, one has to conclude that the ICD is probably more appropriate in international settings, since the WHO makes a considerable effort to ensure international representation on task teams undertaking revision of that system, and also conducts field trials in both HICs and LMICs. However, both the ICD and DSM share a classification paradigm based on Western scientific thought and medical science, and a categorical approach to descriptive psychopathology. A review of the cross-cultural validity of these classification systems is beyond the scope of this article. Suffice it to say that neither the in-process ICD nor the just-completed DSM revisions (with its so-called 'cultural formulation') are likely to change the fact that these hegemonous systems remain inadequate in many cultural contexts.

Finally, it is important to consider the economic implications of expanding medicalisation in psychiatry, especially within poorly resourced contexts. The move towards a dimensional system of classification is likely to have an impact on the epidemiology of mental disorders, and one would expect that the prevalence of mental disorders is likely to increase as more individuals fulfill criteria for diagnosis, where previously their symptoms fell below the threshold. Allen Frances bemoans the fact that inappropriate psychiatric medicalisation of people in the USA is serving to exacerbate a situation where mental health budgets have been severely slashed and access to care has reduced for those with severe mental disorders. ${ }^{[16}$ Frances states: 'The investments in psychiatry are badly misallocated, with excessive diagnosis and treatment for many mildly ill or essentially normal people (who may be more harmed than helped by it), and relative neglect of those with clear psychiatric illness.' He notes that only one-third of people with severe depression get any mental healthcare in the USA. ${ }^{[16]}$ Referring to that context, Frances says: 'This disparity between treatment need and treatment delivery is about to get much worse. ${ }^{\text {[16] }}$ In LMICs, however, the treatment gap is far worse, and it is pertinent to consider the impact of increasing psychiatric medicalisation on this treatment gap. In SA, for example, ZAR70 billion (83\%) of the country's total health expenditure of ZAR84 billion a year is spent in the private sector, which caters for only $15 \%$ of the population. ${ }^{[23]}$ One can reasonably anticipate that increasing the diagnosis and treatment of mildly ill or essentially normal people within the well-resourced private sector, due to expanding psychiatric medicalisation by our diagnostic systems, will result in further widening of the treatment gap. This in turn will serve to worsen the existing disparities in mental healthcare between what is available for the affluent and what is available for the poor. In a country such as SA, rated by the World Bank as one of the most inequitable in the world with a current Gini coefficient of 0.63 , this is a trend that is deeply worrying Thus, the move towards dimensionality and increasing medicalisation has major social, economic and ethical implications that have a bearing on issues of distributive justice, especially in resource-limited contexts. This is a human rights issue and, as a profession, psychiatrists should be conscious of and concerned with the potential global effects of decisions made regarding the definitions of health and disease.

\section{GMH and the need for critical self-reflection in psychiatry}

Recent decades have witnessed the emergence of vigorous global health and GMH movements, with increasing numbers of GMH programmes developing at premiere medical schools, and research funding institutions such as the National Institute of Mental Health in the USA and the Wellcome Trust in the UK formulating new strategies and funding mechanisms focused on GMH initiatives. By definition, the GMH agenda requires a broadened view of mental health that is usually based on forging collaborations between HIC and LMIC partners. While such relationships may benefit LMIC partners through capacity building and foreign funding, and equally benefit HIC partners through furthering their global health agenda, it is important for the HIC parties to recognise and accept certain responsibilities. One such responsibility is to be sensitive to the issue of psychiatric classification systems and their cross-cultural utility within the LMIC context in which they are working. Beyond the common issues of adaptation and translation of methods, instruments and general research strategy to developing, low-resourced contexts, it is arguably a responsibility of the dominant HIC partners to be critically self-reflective of all aspects of the modern psychiatric paradigm that has effectively evolved within the Western North American and European regions. This author contends that a core ethical premise of GMH should be a commitment to engaging with LMIC partners and their environment in a humble, receptive manner that is open to learning about the challenges and difficulties often experienced in practising modern psychiatry, and especially its nosology, within very different sociopolitical, economic and cultural contexts.

Being truly self-reflective in GMH may include asking some of the following questions in relation to psychiatric classification and its ongoing metamorphosis: Whose agenda is the move towards dimensionality? Does it reflect true science or is it driven by the pharmaceutical industry wanting more customers? Is there a political agenda that suits the USA context, allowing more people to obtain benefits and insurance, without thought about how such changes might affect poorly resourced contexts? For example, within some LMIC countries, could such changes in classification provide powerful new political means of pathologising and labelling citizens and extending social control? The move to extend the dimensional approach or even to move towards precision medicine based on biobehavioural phenotypes ${ }^{[24]}$ may be entirely sound in scientific terms and may well benefit patients of the future. But these developments are likely to bring with them a whole range of new ethical dilemmas, not just in LMIC regions but also in HICs. It would be naive to believe that a desire to increase the credibility of psychiatry - as a valid medical discipline based firmly on biological science - is not an important aspect of the current classification agenda. Certainly what is clear from this review is that both categorical and dimensional approaches bring with them as many problems as advantages in terms of ethical challenges. The shape and character of a future classification system that better provides for mental health needs is yet to be described. What is clear, however, is that in this era of evolving psychiatric classification, those involved in GMH - both in HICs and LMICs should feel ethically bound to understanding and highlighting to the global profession the various implications of changing nosology for LMIC regions of the world.

Acknowledgements. The author's research is supported by the National Institute of Mental Health (grant number: 1R21MH093296-01) and by a self-initiated grant from the SA Medical Research Council. 
References

1. Regier DA. Dimensional approaches to psychiatric classification: Defining the research agenda for DSM-V: An introduction. Int J Methods Psychiatr Res 2007;16(Suppl 1):S1-5. [http://dx.doi.org/10.1002/mpr.209]

2. First MB, Westen D. Classification for clinical practice: How to make ICD and DSM better able to serve clinicians. Int Rev Psychiatry 2007;19(5):473-481. [http://dx.doi. org/10.1080/09540260701563429

3. Saxena S, Thornicroft G, Knapp M, Whiteford H. Resources for mental health: Scarcity, inequity, and inefficiency. Lancet 2007;370(9590):878-889. [http://dx.doi.org/10.1016/S0140-6736(07)61239-2]

4. Tsuang MT, van Os J, Tandon R, et al. Attenuated psychosis syndrome in DSM-5. Schizophr Res 2013;150(1):31-35. [http://dx.doi.org/10.1016/j.schres.2013.05.004]

5. Marshall M, Rathbone J. Early intervention for psychosis. Schizophr Bulletin 2011;37(6):1111 1114. [http:/dx.doi.org/10.1093/schbul/sbr110]

6. Allardyce J, Suppes T, van Os J. Dimensions and the psychosis phenotype. Int J Methods Psychiatr Res 2007;16(Suppl 1):S34-40. [http://dx.doi.org/10.1002/mpr.214]

7. Patil T, Giordano J. On the ontological assumptions of the medical model of psychiatry Philosophical considerations and pragmatic tasks. Philos Ethics Humanit Med 2010;28(5):3 [http://dx.doi.org/10.1186/1747-5341-5-3]

8. Cosgrove VE, Suppes T. Informing DSM-5: Biological boundaries between bipolar 1 disorder schizoaffective disorder, and schizophrenia. BMC Medicine 2013;11:127. [http://dx.doi. org/10.1186/1741-7015-11-127]

9. Duval C, Bejanin A, Piolino P, et al. Theory of mind impairments in patients with semantic dementia. Brain 2012;135(1):228-241. [http://dx.doi.org/10.1093/brain/awr309]

10. Kendler KS. A history of the DSM-5 Scientific Review Committee. Psychol Med 2013;43(9):1793-1800. [http://dx.doi.org/10.1017/S0033291713001578]

11. American Psychiatric Association. Diagnostic and Statistical Manual of Mental Disorders, 5th ed. (DSM-5). Washington DC; American Psychiatric Publishing, 2013.

12. First MB. Harmonization of ICD-11 and DSM-5: Opportunities and challenges. $\mathrm{Br}$ Psychiatry 2009;195(5):382-390. [http://dx.doi.org/10.1192/bjp.bp.108.060822]
13. Verdoux H, van Os J. Psychotic symptoms in non-clinical populations and the continuum of psychosis. Schizophr Res 2002;54(1-2):59-65. [http://dx.doi.org/10.1016/S09209964(01)00352-8]

14. Ben-Zeev D, Young MA, Corrigan PW. DSM-V and the stigma of mental illness. J Ment Health 2010;19(4):318-327. [http://dx.doi.org/10.3109/09638237.2010.492484]

15. American Psychological Association Division. Open letter to the DSM-5. http://www. ipetitions.com/petition/dsm5/ (accessed 227 Nov 2013).

16. Frances A. The past, present and future of psychiatric diagnosis. World Psychiatry 2013;12(2):111-112. [http://dx.doi.org/10.1002/wps.20027]

17. Kessler RC, Berglund P, Demler O, et al. Lifetime prevalence and age-of-onset distribution of DSM-IV disorders in the national comorbidity survey replication. Arch Gen Psychiatry 2005;62 (6):593-602. [http://dx.doi.org/10.1001/archpsyc.62.6.593]

18. Burns JK. The ethics of the pharmaceutical industry alliance with psychiatry: A developing country perspective. Journal of Ethics in Mental Health 2009;4(1,Suppl):4e,1-5.

19. Kawa S, Giordano J. A brief historicity of the Diagnostic and Statistical Manual of Menta Disorders: Issues and implications for the future of psychiatric canon and practice. Philos Ethics Humanit Med 2012;7:2. [http://dx.doi.org/10.1186/1747-5341-7-2]

20. Lane C. How shyness became an illness: A brief history of social phobia. In Symposium Unsocial Thought, Uncommon Lives, Common Knowledge, Volume 12, 2006. Durham: Duke University Press, 2006.

21. Jacob KS, Sharan P, Mirza I, et al. Mental health systems in countries: Where are we now? Lancet 2007;370(9592):1061-1077. [http://dx.doi.org/10.1016/S0140-6736(07)61241-0]

22. Fuller Torrey E. Out of the shadows: Confronting America's mental illness crisis. New York Wiley, 2007.

23. Mail \& Guardian. Motsoaledi speaks of 'brutal' private health system. http://mg.co.za/ article/2011-10-09-motsoaledi-speaks-of-brutal-private-health-system (accessed 27 Nov 2013).

24. Cuthbert BN, Insel TR. Toward the future of psychiatric diagnosis: The seven pillars of RDoC BMC Med 2013;11:126. [http://dx.doi.org/10.1186/1741-7015-11-126] 